\title{
COMPLETELY REDUCIBLE LIE ALGEBRAS OF LINEAR TRANSFORMATIONS
}

NATHAN JACOBSON

W. W. Morozov [10] and [11 $]^{1}$ has announced and indicated proofs of the following theorems.

If $\mathfrak{R}$ is a semi-simple Lie algebra over the field of complex numbers, then any element $d$ of $\mathbb{R}$ such that $\operatorname{Ad} d$ is nilpotent can be imbedded in a three-dimensional simple subalgebra.

If $\mathfrak{M}$ is a semi-simple subalgebra of a semi-simple algebra $\mathfrak{R}$ over the field of complex numbers, then the centralizer $\mathfrak{N}$ of $\mathfrak{M}$ in $\mathfrak{l}$ is a direct sum of a semi-simple algebra and its center. Moreover, the elements of the center have adjoint mappings that have simple elementary divisors.

The proof of the first result appears to have a gap ${ }^{2}$ and the proof of the second result is sketched only for the case $\mathfrak{M}$ a three-dimensional simple algebra. In the present note we shall give simple and complete proofs of these results for arbitrary base fields of characteristic 0 . Moreover, we shall give these results what appears to be their natural setting, namely, the theory of completely reducible Lie algebras of linear transformations. We shall also extend Morozov's first result to Lie triple systems (Lemma 4) and we shall use this extension to obtain an analogue of this result for Jordan algebras (Theorem 8).

1. Preliminaries. Throughout this paper we deal exclusively with algebras over fields of characteristic 0 .

Let $\mathfrak{R}$ be a Lie algebra of linear transformations that is completely reducible in the sense that the vector space is completely reducible relative to $\mathfrak{R}$. As is well known, $\mathbb{R}$ is completely reducible if and only if the enveloping associative algebra $\mathbb{E}$ of $\mathfrak{R}$ is semi-simple. In an earlier paper [6] we proved that if $\mathscr{R}$ is completely reducible, then $\mathfrak{R}=\mathfrak{R}_{1} \oplus \mathbb{C}$ where $\mathfrak{Z}_{1}$ is semi-simple and $\mathfrak{C}$ is the center. Also, it is clear that the elements of $\mathbb{S}$ all have simple elementary divisors, since otherwise the enveloping algebra of $\mathbb{E}$ has nilpotent elements and $\&$ has a radical. Conversely suppose that $\mathfrak{R}=\mathfrak{R}_{1} \oplus \mathbb{S}$ where $\mathfrak{R}_{1}$ is semisimple and $\mathfrak{E}$ is the center and consists of elements with simple elementary divisors. Then the enveloping algebra of $\mathbb{R}$ is a homomorphic

Received by the editors December 2, 1949.

${ }^{1}$ Numbers in brackets refer to the bibliography at the end of the paper.

2 The statement "then $x_{0}$ belongs to the zero root, etc." on p. $85,1.8$ of [10] does not seem to be substantiated. 
image of the direct product $\mathfrak{B}$ of the enveloping algebras of $\ell_{1}$ and of the enveloping algebras of the elements of 5 . By the theorem of complete reducibility of the representations of a semi-simple algebra, $\mathfrak{R}_{1}$ is completely reducible and hence its enveloping algebra is semisimple. Hence, $\mathfrak{B}$ is semi-simple and consequently $\mathfrak{R}$ is completely reducible. This proves the following result.

Theorem 1. A necessary and sufficient condition that a Lie algebra of linear transformations be completely reducible is that $\mathbb{R}=\mathfrak{R}_{1} \oplus \mathfrak{S}$ where $\mathfrak{R}_{1}$ is semi-simple and $\mathfrak{S}$ is the center and consists of elements with simple elementary divisors.

We shall also require the following extension of Theorem 3 of the paper referred to in [6].

THEOREM 2. Let $\&$ be a Lie algebra of linear transformations with enveloping associative algebra $\mathbb{E}$. If $\Re$ is the radical of $\mathbb{E}$ and $\mathfrak{S}$ that of $\mathfrak{R}$, then $[\mathbb{R S}] \subseteq \Re$.

Proof. Consider $\xi^{*}=(/ \Re$. Evidently $(\mathfrak{R}+\Re) / \Re$ is a Lie subalgebra of $\mathfrak{F}^{*}$ with $\mathfrak{F}^{*}$ as enveloping algebra. Since $\mathfrak{F}^{*}$ is semi-simple, $(\mathfrak{R}+\Re) / \Re=\left(\mathfrak{R}_{1} / \Re\right) \oplus(\mathfrak{U} / \mathfrak{R})$ where $\mathfrak{R}_{1} / \mathfrak{R}$ is semi-simple and $\mathfrak{U} / \mathfrak{R}$ is the center of $(\mathfrak{R}+\Re) / \Re$. Now

$$
(\mathfrak{R}+\Re) / \mathfrak{U} \cong((\mathfrak{R}+\mathfrak{R}) / \mathfrak{R}) /(\mathfrak{U} / \mathfrak{R}) \cong \mathbb{R}_{1} / \mathfrak{R}
$$

is semi-simple. Since $(\mathfrak{S}+\mathfrak{U}) / \mathfrak{U}$ is a solvable ideal in $(\mathfrak{Q}+\mathfrak{U}) / \mathfrak{U}$, $(\Im+\mathfrak{U}) / \mathfrak{U}=0$ so that $\subseteq \subseteq \mathfrak{U}$. On the other hand, $[(\mathfrak{Q}+\Re) / \Re, \mathfrak{U} / \Re]=0$ and this implies that $[\mathfrak{R U}] \subseteq \Re$. Hence, also $[\mathfrak{R S}] \subseteq \Re$.

REMARKs. By Levi's theorem $\mathfrak{l}$ can be decomposed as $\mathfrak{R}=\mathfrak{S}+\mathfrak{M}$ where $\mathfrak{M}$ is semi-simple and $\mathfrak{S} \cap \mathfrak{M}=0$. It follows that the derived algebra $\mathfrak{R}^{\prime} \equiv[\mathbb{R R}]=[\mathbb{R S}]+[\mathfrak{M M}]$ where $[\mathbb{R S}] \subseteq S$ and $[\mathfrak{M M}] \subseteq \mathfrak{M}$. Hence $\mathbb{R}^{\prime} \cap \mathfrak{S}=[\mathbb{R S}]$. Thus the conclusion of Theorem 2 can be replaced by the following stronger result: $\mathbb{R}^{\prime} \cap \subseteq \subseteq \Re .^{3}$ Since the radical

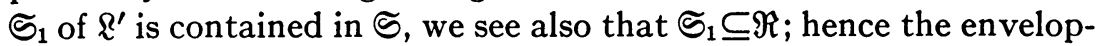
ing algebra of $\widetilde{S}_{1}$ is nilpotent. If we apply this result to the adjoint representation, we obtain a simple proof of a theorem of Cartan $[2$, p. 108] that the radical of the derived algebra of an arbitrary Lie algebra is nilpotent. The condition $\mathbb{R}=\mathfrak{R}_{1} \oplus \mathbb{C}, \mathfrak{R}_{1}$ semi-simple is equivalent to $\mathfrak{I}^{\prime}$ semi-simple. This follows from Levi's theorem and the fact that a semi-simple algebra coincides with its derived algebra.

\section{Some lemmas.}

LEMMA 1. Let $\mathfrak{l}$ be an arbitrary Lie algebra and suppose that $d$ and $h$

\footnotetext{
${ }^{3}$ This remark is due to Chevalley.
} 
are elements of $\mathfrak{R}$ such that $[d h]=d$ and $h \in[\mathfrak{R} d]$. Then there exists another element $e$ in $\mathfrak{R}$ such that $[10$, p. 85]

$$
[d h]=d, \quad[e h]=-e, \quad[d e]=h .
$$

Proof. There exists a $z$ in $\mathfrak{R}$ such that $[z d]=h$. Let $D, Z, H$ be the adjoint mappings of $d, z, h$ respectively. Then

$$
[D H]=D, \quad[Z D]=H .
$$

Because of the first of these equations $D$ is nilpotent [6, Lemma 2]. Also,

$$
\begin{aligned}
{[[z h]+z, d] } & =[[z d] h]+[z[h d]]+[z d] \\
& =0-[z d]+[z d]=0 .
\end{aligned}
$$

Hence, $[z h]=-z+x_{1}$ where $x_{1} \in \Omega_{1}$ the subalgebra of elements that commute with the element $d$. Since $[D H]=D, \Omega_{1} H \subseteq \Omega_{1}$. Also

$$
\begin{aligned}
{\left[Z D^{i}\right] } & =H D^{i-1}+D H D^{i-1}+\cdots+D^{i-1} H \\
& =D^{i-1}(H-(i-1))+D^{i-1}(H-(i-2))+\cdots+D^{i-1} H \\
& =i D^{i-1}\left(H-\frac{i-1}{2}\right) .
\end{aligned}
$$

Now let $b=a D^{i-1} \in \Omega_{1} \cap R D^{i-1}$. Then $a D^{i}=0$ and

$$
\begin{aligned}
i\left(a D^{i-1}\right)\left(H-\frac{i-1}{2}\right) & =a Z D^{i}-a D^{i} Z \\
& =(a Z) D^{i} .
\end{aligned}
$$

Hence $b(H-(i-1) / 2) \equiv 0\left(\bmod \Re_{1} \cap \Omega D^{i}\right)$. It follows that $H+1$ is a 1-1 linear transformation of $\Omega_{1}$ onto itself. Hence we can find a $y_{1} \in \Omega_{1}$ such that $y_{1}(H+1)=-x_{1}$, that is, $\left[y_{1} h\right]+y_{1}=-x_{1}$. Then

$$
\begin{aligned}
{\left[z+y_{1}, h\right] } & =[z h]+\left[y_{1} h\right] \\
& =-z+x_{1}-y_{1}-x_{1}=-z-y_{1} .
\end{aligned}
$$

It follows that $e=-z-y_{1}$ satisfies

$$
[e h]=-e, \quad[d h]=d, \quad[d e]=h .
$$

LEMMA 2. If $d$ is a nilpotent matrix in the matrix algebra $\Phi_{n}, \Phi a$ field, then there exist matrices $h$ and e such that (1) holds.

Proof. We use induction on $n$. If $d$ is decomposable, we get a reduction to matrices of lower degree. Hence, we may suppose that $d=e_{12}+e_{23}+\cdots+e_{n-1, n}$. Then 


$$
\begin{aligned}
h= & -\frac{n-1}{2} e_{11}+\left(1-\frac{n-1}{2}\right) e_{22}+\left(2-\frac{n-1}{2}\right) e_{33}+\cdots \\
& +\left(n-1-\frac{n-1}{2}\right) e_{n, n}, \\
e= & -\frac{n-1}{2} e_{21}+\left(1-\frac{2(n-1)}{2}\right) e_{32}+\cdots \\
& +\left(\frac{(i-1)(i-2)}{2}-\frac{(i-1)(n-1)}{2}\right) e_{i, i-1}+\cdots \\
& +\left(\frac{(n-1)(n-2)}{2}-\frac{(n-1)^{2}}{2}\right) e_{n, n-1}
\end{aligned}
$$

satisfies the given conditions.

LEMMA 3. Let $\mathbb{R}$ be a Lie algebra of linear transformations that has the property that any nilpotent element $d$ can be imbedded in a three-dimensional simple subalgebra $(d, e, h)$ such that (1) holds. Then any subalgebra $\mathfrak{M}$ that has a complementary subspace $\mathfrak{U}$ of $\mathfrak{Q}$ invariant relative to $\mathfrak{M}$ has the property stated for $\mathbb{R}$.

Proof. Our assumption is that $\mathfrak{l}=\mathfrak{M} \oplus \mathfrak{U}$ where $[\mathfrak{U M}] \subseteq \mathfrak{U}$. If $u$ is an element of $\mathfrak{M}$ which is nilpotent, then we can find a $v$ and a $w$ in $\mathfrak{R}$ such that $[u w]=u,[v w]=-v,[u v]=w$. Write $w=w_{1}+w_{2}, w_{1}$ in $\mathfrak{u}$, $w_{2}$ in $\mathfrak{M}, v=v_{1}+v_{2}, v_{1}$ in $\mathfrak{u}, v_{2}$ in $\mathfrak{M}$. Then $\left[u w_{1}\right]+\left[u w_{2}\right]=u$. Hence $\left[u w_{2}\right]=u$. Also $\left[u v_{1}\right]+\left[u v_{2}\right]=w_{1}+w_{2}$, so that $\left[u v_{2}\right]=w_{2}$. Thus $w_{2}$ $\in[\{u]$. Hence by Lemma 1 we can find a $c$ in $\&$ such that

$$
\left[u w_{2}\right]=u, \quad\left[c w_{2}\right]=-c, \quad[u c]=w_{2} .
$$

Write $c=c_{1}+c_{2}$. Then

$$
\left[u c_{2}\right]=w_{2}, \quad\left[c_{2} w_{2}\right]=-c_{2} .
$$

Hence $\left(u, c_{2}, w_{2}\right)$ is a three-dimensional simple subalgebra of $\mathfrak{M}$ containing $u$.

3. Imbedding of nilpotent elements in three-dimensional simple subalgebras. We can now prove the following theorem.

THEOREM 3. If $\mathfrak{R}$ is a completely reducible Lie algebra of linear transformations, any nilpotent element $d$ of $\mathfrak{R}$ can be imbedded in a threedimensional simple subalgebra of $\mathbb{R}$.

Proof. We know that $\mathfrak{l}=\mathfrak{R}_{1} \oplus \mathfrak{C}$ where $\mathfrak{R}_{1}$ is semi-simple and $\mathfrak{C}$ is the center and all the elements of $\mathbb{S}$ have simple elementary divisors. Let $\Omega$ be the complete algebra of linear transformations acting in the 
same space as $\mathfrak{R}$. Let $\mathfrak{R}^{*}, \mathfrak{R}_{1}^{*}$, $\mathfrak{C}^{*}$ be the sets of adjoint mappings acting in $\Omega$ and determined by the subalgebras $R, \Omega_{1}$, $\mathbb{S}$ respectively. Since $\Omega_{1}^{*}$ is semi-simple, it is completely reducible. Moreover the elements of (5* all have simple elementary divisors. Hence this commutative set of linear transformations is also completely reducible. Since $\left[\mathbb{R}_{1}^{*}, \mathfrak{C}^{*}\right]$ $=0$, it follows that $\Omega^{*}=\Omega_{1}^{*}+\mathfrak{C}^{*}$ is a completely reducible set of linear transformations. Thus we can decompose $\Re$ as $\mathfrak{Q} \oplus \mathfrak{M}$ where [MR] $\subseteq \mathfrak{M}$. Since $\Omega$ is isomorphic to a complete matrix algebra, it follows from Lemmas 2 and 3 that $d$ can be imbedded in a three-dimensional simple subalgebra of $\mathfrak{R}$.

We recall that a linear transformation $a$ can be expressed in one and only one way as a sum of two commuting linear transformations $s$ and $n$ where $s$ has simple elementary divisors and $n$ is nilpotent. (Cf. $[3$, p. 523].) These linear transformations are polynomials in $a$. Hence they commute with every linear transformation commutative with $a$. Following Malcev [9] we say that a Lie algebra $\mathfrak{l}$ of linear transformations is splittable if the parts $s$ and $n$ of every $a \in \mathbb{R}$ also belong to $\mathfrak{R}$. It is clear that the center of a splittable Lie algebra is splittable. We can use this concept to obtain a partial converse of Theorem 3.

TheOREM 4. Let $\mathfrak{R}$ be a Lie algebra of linear transformations such that (1) every nilpotent element of $\mathbb{R}$ is contained in a three-dimensional simple subalgebra, (2) the center of $\mathbb{R}$ is splittable. Then $\mathbb{R}$ is completely reducible.

Proof. Consider a Levi decomposition $\mathfrak{l}=\mathfrak{R}_{1} \oplus \mathfrak{S}$ where $\mathfrak{R}_{1}$ is semisimple and $\mathfrak{S}$ is the radical. If $d \in$ [RS], Theorem 2 shows that $d$ is nilpotent. Hence $d$ can be imbedded in a three-dimensional subalgebra $\mathfrak{B}$ of $\mathfrak{R}$. If $d \neq 0$, it follows that $\subseteq \supseteq \mathfrak{B}$. Since $\subseteq$ is solvable, this is impossible. Hence $d=0$. Thus [RS] $=0$ and $\subseteq=\mathfrak{S}$ is the center. A similar argument shows that $\mathbb{S}$ contains no nilpotent elements not equal to 0 . Since $\mathbb{S}$ is splittable, it follows that all the elements of $\mathfrak{S}$ have simple elementary divisors. Hence by Theorem $1, \mathbb{R}$ is completely reducible.

4. Centralizers of completely reducible Lie algebras. We shall prove first the following criterion that a subalgebra of a completely reducible Lie algebra of linear transformations be completely reducible.

THEOREM 5. Let $\mathfrak{R}$ be a completely reducible Lie algebra and let $\mathfrak{M}$ be a subalgebra such that (1) $\mathfrak{M}$ has a complementary subspace in $\mathfrak{R}$ invariant under $\mathfrak{M}$, (2) the center of $\mathfrak{M}$ is splittable. Then $\mathfrak{M}$ is completely reducible. 
This is immediate from Lemma 3, Theorems 3 and 4.

We require also the following result.

TheOREM 6. Any completely reducible algebra of linear transformations is splittable.

Proof. This result can be deduced from the theory of algebraic Lie algebras [4], or $[5$, p. 37]. However, we shall give an independent demonstration. It is easy to see from Theorem 1 that it suffices to prove our result for semi-simple algebras. Hence, let $\mathbb{R}$ be semisimple. Let $a \in \mathbb{R}$ and write $a=s+n$ where $[s n]=0$ and $s$ has simple elementary divisors and $n$ is nilpotent. Let $A, S, N$ be the adjoint mappings of the complete algebra of linear transformations determined by the elements $a, s, n$ respectively. Then $A=S+N$, $[S N]=0, S$ has simple elementary divisors, $N$ nilpotent. Hence $S$ and $N$ are polynomials in $A$ and consequently the relation $R A \subseteq R$ implies $\mathbb{R} \subseteq \subseteq \mathbb{R}$ and $\mathbb{R} N \subseteq \mathfrak{R}$. Hence $S$ and $N$ are derivations in $\mathfrak{R}$. Since the derivations of $\mathbb{R}$ are all inner, there exist elements $s_{1}, n_{1}$ in $\mathbb{R}$ such that

$$
[x s]=\left[x s_{1}\right] \text { and }[x n]=\left[x n_{1}\right]
$$

for all $x$ in $\mathbb{R}$. We can assume that the matrices of $\mathfrak{R}$ have the reduced form

$$
\operatorname{diag}\left\{a_{1}, a_{2}, \cdots, a_{8}\right\}
$$

where the $a_{i}$ have elements in the algebraic closure $\Omega$ of the base field and for each $i$ the set of matrices $\left\{a_{i}\right\}$ is irreducible in $\Omega$. Since $n$ is a polynomial in $a$, its matrix has the form (3) also. Hence $n-n_{1}$ has this form. Since $n-n_{1}$ commutes with every $x$ in $\&$, we can use Schur's lemma to prove that the matrix of $n-n_{1}$ is

$$
\operatorname{diag}\left\{\nu_{1}, \nu_{2}, \cdots, \nu_{s}\right\}
$$

where each $\nu_{i}$ is a scalar. On the other hand, $n$ is nilpotent and $n_{1} \in \mathbb{R}=[\mathbb{R}]$, and this implies that $\operatorname{tr} \nu_{i}=0$ for $i=1,2, \cdots, s$. Hence $\nu_{i}=0$ and $n=n_{1} \in \mathbb{R}$. Hence, also, $s \in \mathbb{R}$.

We are now in a position to prove the following result.

TheOREM 7. If $\mathfrak{M}$ is a completely reducible subalgebra of a completely reducible Lie algebra $\mathfrak{R}$, then the centralizer $\mathfrak{N}$ of $\mathfrak{M}$ in $\mathfrak{R}$ is completely reducible.

Proof. Since $\mathfrak{R}$ is splittable, so is $\mathfrak{R}$, and so also is the center of $\mathfrak{N}$. Hence in view of Theorem 5 it suffices to show that $\mathfrak{N}$ has a complementary subspace in $\mathfrak{R}$ invariant relative to $\mathfrak{R}$. Write $\mathfrak{M}=\mathfrak{M}_{1} \oplus \mathfrak{C}$ where $\mathfrak{M}_{1}$ is semi-simple and $\mathfrak{C}$ is the center and consists of trans- 
formations with simple elementary divisors. Now the adjoints in $\mathfrak{Z}$ of the elements of $\mathbb{S}$ have simple elementary divisors. It follows that the set $\mathfrak{M}^{*}$ of adjoints of the elements of $\mathfrak{M}$ is completely reducible. Hence, $\mathfrak{l}=\mathfrak{M}_{1} \oplus \mathfrak{M}_{2} \oplus \cdots \oplus \mathfrak{M}_{h}$ where the $\mathfrak{M}_{i}$ are $\mathfrak{M}$-invariant and irreducible. Let $\mathfrak{M}_{1}, \mathfrak{M}_{2}, \cdots, \mathfrak{M}_{k}$ be the $\mathfrak{M}_{i}$ such that $\left[\mathfrak{M}_{i} \mathfrak{M}\right]=0$. Then

$$
\mathfrak{N}=\mathfrak{M}_{1}+\mathfrak{M}_{2}+\cdots+\mathfrak{M}_{k} \text {. }
$$

If $j>k$,

$$
\mathfrak{M}_{j}=\left[\mathfrak{M}_{j} \mathfrak{M}\right]+[[\mathfrak{M} ; \mathfrak{M}] \mathfrak{M}]+\cdots
$$

Hence, if we set $\mathfrak{U}=\mathfrak{M}_{k+1}+\mathfrak{M}_{k+2}+\cdots+\mathfrak{M}_{h}$, then

$$
\mathfrak{U}=[\mathfrak{R M}]+[[\mathfrak{R M}] \mathfrak{M}]+\cdots
$$

and $\mathfrak{R}=\mathfrak{N} \oplus \mathfrak{U}$. Also

$$
\begin{aligned}
{[[\cdots[[\mathbb{M}] \mathfrak{M}] \cdots \mathfrak{M}] \mathfrak{R}] } & \subseteq[[\cdots[\mathfrak{R N}] \mathfrak{M}] \cdots \mathfrak{M}] \\
& \subseteq[[[\mathbb{R}] \mathfrak{M}] \cdots \mathfrak{M}]
\end{aligned}
$$

so that $[\mathfrak{U} \mathfrak{N}] \subseteq \mathfrak{U}$. Thus $\mathfrak{U}$ is a complementary subspace invariant relative to $\mathfrak{N}$. This completes the proof.

5. Application to Jordan algebras. We suppose first that $\mathfrak{U}$ is a Lie triple system of linear transformations, that is, $\mathfrak{U}$ is subspace of linear transformations closed relative to the ternary composition $[a b c] \equiv[[a b] c][7]$. If $\mathfrak{U}$ is a Lie triple system, then $[\mathfrak{u} \mathfrak{u}]$ is a Lie algebra and $\mathfrak{u}+[\mathfrak{u} \mathfrak{u}]$ is a Lie algebra. The latter is the enveloping Lie algebra of $\mathfrak{U}$.

LеммA 4. Let $\mathfrak{u}$ be a completely reducible Lie triple system such that $\mathfrak{u} \cap[\mathfrak{u} \mathfrak{U}]=0$. Then if $d$ is a nilpotent element of $\mathfrak{u}$ there exists a second nilpotent element $e$ in $\mathfrak{U}$ such that $[e d d]=d,[$ dee $]=e$.

Proof. $\mathfrak{R}=\mathfrak{U} \oplus[\mathfrak{u} \mathfrak{U}]$ is a completely reducible Lie algebra. Hence, we can find $b, c$ in $\mathfrak{R}$ such that $[d c]=d,[b c]=-b,[d b]=c$. Write $c=c_{1}+c_{2}, c_{1}$ in $\mathfrak{U}, c_{2}$ in [ $[\mathfrak{u} \mathfrak{U}], b=b_{1}+b_{2}, b_{1}$ in $\mathfrak{U}$ and $b_{2}$ in [ $\left.\mathfrak{u} \mathfrak{U}\right]$. Then $\left[d c_{2}\right]=d,\left[d b_{1}\right]=c_{2}$. Hence by Lemma 1 we can find an element $f$ in $\mathfrak{Z}$ such that $\left[d c_{2}\right]=d,\left[f c_{2}\right]=-f,[d f]=c_{2}$. Set $f=f_{1}+f_{2}, f_{1}$ in $\mathfrak{u}, f_{2}$ in $[\mathfrak{u} \mathfrak{u}]$. Then $\left[f_{1} c_{2}\right]=-f_{1}$ and $\left[d f_{1}\right]=c_{2}$. It follows that $e=f_{1}$ satisfies the required conditions.

LEMMA 5. If $d$ and $e$ are nonzero linear transformations such that $[e d d]=d,[d e e]=e$, then the transformations $d \pm e$ are not nilpotent.

Proof. If we set $h=[d e]$, then $(d, e, h)$ satisfies (1). It is known 
that in the irreducible representation of degree $s+1$ of the Lie algebra $(d, e, h)$ we can take the representing matrices to be

$$
\begin{aligned}
h= & -\frac{s}{2} e_{11}+\left(1-\frac{s}{2}\right) e_{22}+\cdots+\left(\frac{s}{2}\right) e_{s+1,8+1}, \\
d= & e_{12}+e_{28}+\cdots+e_{s, 8+1}, \\
e= & -\frac{s}{2} e_{21}+\cdots+\left(\frac{(i-1)(i-2)}{2}-\frac{(i-1) s}{2}\right) e_{i, i-1}+\cdots \\
& +\left(\frac{-s}{2}\right) e_{s+1,8}
\end{aligned}
$$

It follows that

$$
\operatorname{tr} d e=-\frac{1}{2} \sum_{i=2}^{s+1}(i-1)(s+2-i) \geqq 0
$$

in the irreducible representation of degree $s+1$. Hence if $d$ and $e$ have their original meanings, then $\operatorname{tr} d e \neq 0$. Since $d$ and $e$ are nilpotent, $\operatorname{tr} d^{2}=\operatorname{tr} e^{2}=0$. Hence $\operatorname{tr}(d \pm e)^{2} \neq 0$ and $d \pm e$ is not nilpotent.

Now let $\mathfrak{A}$ be a Jordan algebra, that is, $\mathfrak{A}$ is a commutative algebra in which the identity $(x a) a^{2}=\left(x a^{2}\right) a$ holds. Let $\mathfrak{U}$ be the set of (right) multiplications $R_{a}: x \rightarrow x a$. We have the fundamental relation due to Albert [8] and [1] that $\left[R_{c} R_{b} R_{a}=R_{a\left[R_{b} R_{c}\right]}\right]$ which shows that $\mathfrak{U}$ is a Lie triple system. If $\mathfrak{A}$ has an identity, then $\mathfrak{U} \cap[\mathfrak{u} \mathfrak{U}]=0$. If $\mathfrak{A}$ is semi-simple, $\mathfrak{U}$ is completely reducible. We use these facts and the above lemmas to prove the following criterion.

Theorem 8. A Jordan algebra $\mathfrak{A}$ is semi-simple if and only if for every nilpotent element $z$ of $\mathfrak{A}$ there exists a second nilpotent element $w$ in $\mathfrak{A}$ such that

$$
z^{2} w-z(z w)=z, \quad w^{2} z-w(w z)=w .
$$

Proof. An element $z$ of $\mathfrak{A}$ is nilpotent if and only if $R_{z}$ is nilpotent $[1$, Theorem 1]. Hence, if $\mathfrak{A}$ is semi-simple and $z$ is a nilpotent element of $\mathfrak{A}$, then by Lemma 4 there exists a $w$ in $\mathfrak{A}$ such that $R_{w}$ is nilpotent and $\left[R_{w} R_{z} R_{z}\right]=R_{z},\left[R_{z} R_{w} R_{w}\right]=R_{w}$. It follows that $R_{z\left[R_{z} R_{w]}\right.}=R_{z}$ and $z=z\left[R_{z} R_{w}\right]=z^{2} w-z(z w)$. Similarly, $w^{2} z-w(w z)=w$. Conversely,

4 Cf. the proof of Lemma 2. It is well known that any two irreducible representations are equivalent. This can be seen by using the general theory of representations of semi-simple Lie algebras, or it can be established directly by elementary methods. 
suppose that $\mathfrak{A}$ has the property stated in the theorem. Let $z$ be in the radical $\mathfrak{N}$ of $\mathfrak{A}$. Then $z$ is nilpotent. Hence, there exists a $w$ in $\mathfrak{N}$ such that (4) holds. The second of these conditions shows that $w \in \mathfrak{N}$. Now if $z \neq 0$, then $R_{z} \neq 0$. For $R_{z}=0$ implies that $z^{2}=0$ and $z w=0$. Hence, by (4), $z=0$. Also (4) shows that $w \neq 0$ and so $R_{w} \neq 0$. By Lemma $5, R_{z \pm w}$ is not nilpotent and this contradicts the relation $z \pm w \in \mathfrak{N}$. Hence $z=0, \mathfrak{N}=0$, and $\mathfrak{A}$ is semi-simple.

(Added April 27, 1950.) We note finally the following application to the theory of derivations of Jordan algebra.

THEOREM 9. The derivation algebra of any semi-simple Jordan algebra is completely reducible.

Proof. It is known that the derivation algebra $\mathfrak{D}$ is the space [ $\mathfrak{U} \mathfrak{U}]$ where $\mathfrak{U}$ is the space of right multiplication [8]. Thus the subalgebra $\mathfrak{D}$ of the completely reducible Lie algebra $\mathfrak{l}=\mathfrak{U}+[\mathfrak{u} \mathfrak{u}]$ has the complementary space $\mathfrak{U}$ in $\mathfrak{R}$ invariant under $\mathfrak{D}$. Moreover, it is a well known result of Gantmacher that derivation algebras are splittable (cf. [4]). Hence, by Theorem $5, \mathfrak{D}$ is completely reducible.

\section{BIBLIOGRAPHY}

1. A. A. Albert, $A$ structure theory for Jordan algebras, Ann. of Math. vol. 48 (1947) pp. 546-567.

2. E. Cartan, Thèse, Paris, 1894.

3. C. Chevalley, $A$ new kind of relationship between matrices, Amer. J. Math. vol. 65 (1943) pp. 521-531.

4. C. Chevalley and H. F. Tuan, On algebraic Lie algebras, Proc. Nat. Acad. Sci. U.S.A. vol. 31 (1945) pp. 195-196.

5. M. Goto, On algebraic Lie algebras, Journal of the Mathematical Society of Japan vol. 1 (1948) pp. 29-45.

6. N. Jacobson, Rational methods in the theory of Lie algebras, Ann. of Math. vol. 36 (1935) pp. 875-881.

7. —, Lie and Jordan triple systems, Amer. J. Math. vol. 71 (1949) pp. 149170 .

8. - Derivation and multiplication algebras of semi-simple Jordan algebras, Ann. of Math. vol. 50 (1949) pp. 866-874.

9. A. Malcev, On solvable Lie algebras, Bull. Acad. Sci. URSS. Ser. Math. vol. 9 (1945) pp. 329-356.

10. W. W. Morozov, On a nilpotent element of a semi-simple Lie algebra, C. R. (Doklady) Acad. Sci. URSS. vol. 36 (1942) pp. 83-86.

11. - On the centralizer of a semi-simple subalgebra of a semi-simple Lie algebra, ibid. pp. 259-261.

YALE UNIVERSITY 